EPJ Web of Conferences 59, 05019 (2013)

DOI: $10.1051 /$ epjconf/20135905019

(C) Owned by the authors, published by EDP Sciences, 2013

\title{
Number-conserving linear response study of low-velocity ion stopping in a collisional magnetized classical plasma
}

\author{
Hrachya B. Nersisyan ${ }^{1}$ and Claude Deutsch ${ }^{2, a}$ \\ ${ }^{1}$ Plasma Theory Group, Institute of Radiophysics and Electronics, 0203 Ashtarak, Armenia \\ ${ }^{2}$ LPGP (UMR-CNRS 8578), Université Paris XI, 91405 Orsay, France
}

\begin{abstract}
The low-velocity stopping power of ions in a magnetized collisional plasma is studied through the linear response theory. The collisions are taken into account through a number-conserving relaxation time approximation. One of the major objectives of this study is to compare and contrast our theoretical results with those obtained through a diffusion coefficient formulation based on Dufty-Berkovsky relation.
\end{abstract}

\section{INTRODUCTION}

The energy loss of ion beams in magnetized plasmas are important in many areas of physics such as transport and the magnetized target fusions [1], or inertial confinement fusion. The latter presently advocates a highly regarded fast ignition scenario [2], based on femtolaser produced ion beams impinging a precompressed capsule containing a thermonuclear fuel in it. These interaction geometries highlight low ion velocity slowing down (LIVSD) as playing a fundamental role in asserting the confining capabilities and thermonuclear burn efficiency in dense and strongly magnetized media.

In this paper we report on a theoretical study of energy loss of a low-velocity ion in a magnetized plasma through the linear response (LR) approach which conserves particle number. Since the early 1960s, a number of theoretical calculations of the stopping power within LR treatment in a magnetized plasma have been presented (a book [3] summarizes research in this area over the past five decades). In this approach the central quantity which determines the stopping characteristics of a medium is the dielectric function (DF). Our first objective is to derive the DF in a number-conserving manner using the Bhatnagar-Krook-Gross (BGK) approach for a collisional and magnetized classical plasma and using this DF to calculate the energy loss of an ion. A second objective of our study is to contrast our theoretical results with those obtained in Ref. [4] employing the Dufty-Berkovsky (DB) relation [5].

\section{LINEAR RESPONSE FORMULATION}

Within the LR the electron plasma is described by the distribution function $f(\mathbf{r}, \mathbf{v}, t)$ which is determined by the kinetic and Poisson equations. Usually only a mean-field interaction between the electrons is considered and hard collisions are neglected. This is valid for weakly coupled plasmas when $4 \pi n_{0} \lambda^{3}=1 / \epsilon \gg 1$. Here $\epsilon$ is the plasma parameter, $n_{0}$ and $\lambda$ are the equilibrium density and the Debye length of electrons, respectively.

We consider a nonrelativistic projectile ion with charge $Z e$ and with a velocity $\mathbf{v}$, which moves in a magnetized collisional and classical plasma at an angle $\vartheta$ with respect to the constant magnetic field $\mathbf{B}$.

\footnotetext{
ae-mail: claude.deutsch@pgp.u-psud.fr
}

This is an Open Access article distributed under the terms of the Creative Commons Attribution License 2.0, which permits unrestricted use, distribution, and reproduction in any medium, provided the original work is properly cited. 
We ignore any role of the electron spin or magnetic moment due to the nonrelativistic motion of the ion and the plasma electrons. The effect of collisions on the dielectric properties of the plasma is included, in a number-conserving approximation, through a relaxation time $\tau=1 / \gamma$, where $\gamma$ is the collision frequency [6]. Thus we consider the kinetic equation of the collisional plasma within relaxation-time approximation (RTA) in which the collision term is of the BGK-type [6],

$$
\frac{\partial f}{\partial t}+\mathbf{v} \cdot \frac{\partial f}{\partial \mathbf{r}}-\frac{e}{m}\left[\mathbf{E}+\frac{1}{c}[\mathbf{v} \times \mathbf{B}]\right] \cdot \frac{\partial f}{\partial \mathbf{v}}=-\gamma\left[f-\frac{n}{n_{0}} f_{0}(\mathbf{v})\right],
$$

where the collision frequency $\gamma$ is a measure of damping of excitations in the plasma, and $n(\mathbf{r}, t)=$ $\int f(\mathbf{r}, \mathbf{v}, t) d \mathbf{v}, n_{0}=\int f_{0}(\mathbf{v}) d \mathbf{v}$. Here $n(\mathbf{r}, t)$ and $f_{0}(\mathbf{v})$ are the density and the equilibrium distribution function of the electrons. It is easy to see that this form of collision term conserves the total number of particles. $\mathbf{E}=-\nabla \varphi$ is a self-consistent electric field, and $\mathbf{B}$ is treated as an external magnetic field.

For a small perturbations we assume $f=f_{0}+f_{1}$. We solve the system of the linearized kinetic and Poisson equations by space-time Fourier transforms. The general solution of these equations has been obtained in Ref. [7]. In particular, assuming unperturbed Maxwell distribution function we obtain

$$
\begin{gathered}
\varphi(\mathbf{k}, \omega)=\frac{4 \pi \rho_{0}(\mathbf{k}, \omega)}{k^{2} \varepsilon_{M}(\mathbf{k}, \omega, \gamma)}, \\
\varepsilon_{M}(\mathbf{k}, \omega, \gamma)=1+\frac{(\omega+i \gamma)[\varepsilon(\mathbf{k}, \omega, \gamma)-1]}{\omega+i \gamma Q(\mathbf{k}, \omega, \gamma)},
\end{gathered}
$$

where $\varphi$ is the electrostatic potential, $\rho_{0}$ is the density of the external charge and $\varepsilon_{M}(\mathbf{k}, \omega, \gamma)$ is the collision-inclusive DF of the magnetized plasma with $\varepsilon(\mathbf{k}, \omega, \gamma)=1+(k \lambda)^{-2} Q(\mathbf{k}, \omega, \gamma)$ and

$$
Q(\mathbf{k}, \omega, \gamma)=1+(i s-\varsigma) \int_{0}^{\infty} e^{i s t-X(t)-\varsigma t} d t
$$

where $s=\omega / k v_{e}, \varsigma=\gamma / k v_{e}$, and $X(t)=\left(t^{2} / 2\right)\left(k_{\|}^{2} / k^{2}\right)+k_{\perp}^{2} a_{e}^{2}\left[1-\cos \left(t / k a_{e}\right)\right]$. Here $a_{e}=v_{e} / \omega_{c}$, $\omega_{c}=e B / m c$ and $v_{e}$ is the thermal velocity of the electrons. $k_{\|}$and $k_{\perp}$ denote the components of $\mathbf{k}$ parallel or perpendicular to $\mathbf{B}$, respectively. Note that Eq. (3) formally coincides with the Mermin DF [8] derived for a degenerate electron gas (DEG) without magnetic field. In contrast to the collisionless DF, Eq. (3) leads to the Drude behavior as well as the broadening of the energy loss function (ELF) at long wavelengths $(k \rightarrow 0)$ due to the damping compared to the ELF with vanishing damping [7].

\section{LOW-VELOCITY STOPPING POWER}

In this section we consider the stopping power (SP) of a low-velocity ion moving in a magnetized collisional plasma for an arbitrary angle $\vartheta$ with respect to B. The SP $S$ of an ion is defined as the energy loss of the ion in a unit length due to interaction with a plasma. From Eq. (2) it is straightforward to calculate the electric field $\mathbf{E}=-\nabla \varphi$ and the stopping force acting on the ion. Then, the SP of the ion is expressed by the ELF $\operatorname{Im}\left[\epsilon_{M}^{-1}\right]$ at $\omega=\mathbf{k} \cdot \mathbf{v}$ [3]. For the friction coefficient we have to consider $S$ in a low-velocity limit writing the Taylor expansion of Eq. (3) for small $\omega=\mathbf{k} \cdot \mathbf{v}$. Thus we obtain usual (linear with respect to $v$ ) friction law $S(\vartheta) \simeq\left(2 Z^{2} e^{2} / \sqrt{2 \pi} \lambda^{2}\right)\left(v / v_{e}\right) \mathcal{R}(\vartheta)$,

$$
\begin{aligned}
\mathcal{R}(\vartheta)= & \frac{1}{2} \int_{0}^{\infty} e^{-\left(2 k^{2} / \zeta^{2}\right) \sin ^{2}(\zeta t)-2 v t} \frac{d t}{t \Upsilon(t)} \\
& \times \int_{0}^{\kappa} \frac{k^{3} d k}{\left(k^{2}+1\right)^{2}}\left[\Phi_{1}(k Q(t)) \cos ^{2} \vartheta+\frac{1}{2} \Phi_{2}(k Q(t)) \sin ^{2} \vartheta\right],
\end{aligned}
$$



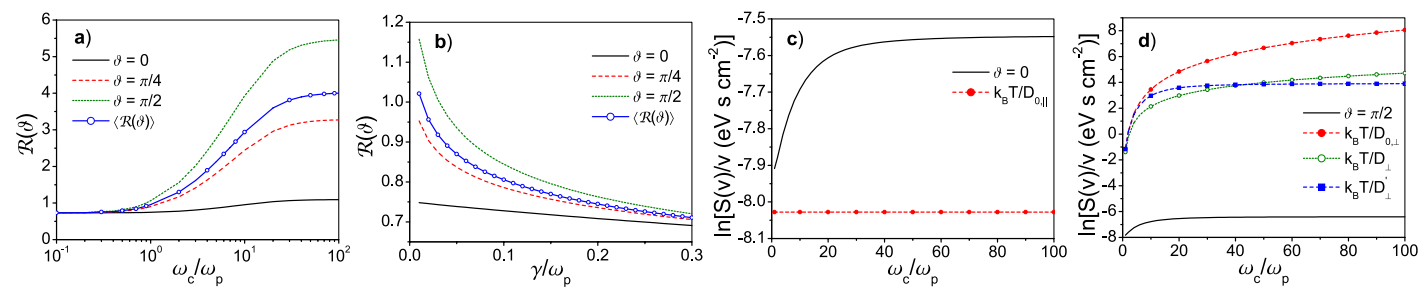

Figure 1. a) and b): The friction coefficient $\mathcal{R}(\vartheta)$ vs $\omega_{c} / \omega_{p}$ (a) and $\gamma / \omega_{p}$ (b) for $\kappa=15, \gamma / \omega_{p}=0.02$ (a), $\omega_{c}=\omega_{p}$ (b). The lines with symbols corresponds to the angular averaged $\langle\mathcal{R}(\vartheta)\rangle$. c) and d): Proton LIVSD in a plasma with $n_{0}=10^{17} \mathrm{~cm}^{-3}, T=1 \mathrm{eV}$ vs $\omega_{c} / \omega_{p}$. The symbols in c) and d) represent parallel and transverse LIVSD, respectively. The solid lines were obtained from Eq. (5).

with $Q(t)=\sqrt{2} t \Upsilon(t)$. Here $\mathcal{R}(\vartheta)$ is the dimensionless friction coefficient (FC), $\kappa=1 / \epsilon|Z| \gg 1$, $v=\gamma / \omega_{p}, \zeta=\omega_{c} / \omega_{p}, \omega_{p}$ is the plasma frequency. $\Upsilon^{2}(t)=1-[\sin (\zeta t) / \zeta t]^{2}, \Phi_{1}(x)=x^{-2} \Phi(x)$, $\Phi_{2}(x)=2 \operatorname{erf}(x)-\Phi_{1}(x)$, and $\Phi(x)=\operatorname{erf}(x)-(2 / \sqrt{\pi}) x e^{-x^{2}}$, where $\operatorname{erf}(z)$ is the error function. At $B \rightarrow 0$ the FC $\mathcal{R}_{0}(\vartheta)$ is isotropic. In addition at $v \rightarrow 0$, we recover the usual low-velocity SP in an unmagnetized collisionless plasma with the FC $\mathcal{R}_{0}(\vartheta)=(1 / 6) U(\kappa)$ with $U(\kappa) \simeq 2 \ln \kappa-1$ at $\kappa \gg 1$ [3]. At $\zeta \rightarrow \infty$, the plasma becomes highly anisotropic. Then the FC at $\gamma \ll \omega_{p}$ becomes

$$
\mathcal{R}_{\infty}(\vartheta)=\frac{1}{4}\left\{\sin ^{2} \vartheta\left[\left(\ln \left(\sqrt{2} \omega_{p} / \gamma\right)-\frac{C+1}{2}\right) U(\kappa)+U_{1}(\kappa)\right]+U(\kappa) \cos ^{2} \vartheta\right\}
$$

where $C=0.5772$ is the Euler's constant, and at $\kappa \gg 1$ (weak ion-plasma coupling) the function $U_{1}(\kappa)$ is approximated by $U_{1}(\kappa) \simeq \ln ^{2} \kappa-\pi^{2} / 12$. It is seen that the first term in Eq. (6) diverges logarithmically at vanishing $\gamma$. It can be shown that the general expression (5) for the FC derived for finite magnetic field behaves similarly. This is a consequence due to the magnetic field since $\mathcal{R}_{0}(\vartheta)$ remains finite as $\gamma \rightarrow 0$. The divergent term in Eq. (6) vanishes, however, when the ion moves along the magnetic field $(\vartheta=0)$. Then the friction coefficient is given by the last term of Eq. (6).

It has been shown by DB [5] that the low-velocity SP of an ion is related to the diffusion coefficient (DC) $D$ through $S(v) /\left.v\right|_{v \rightarrow 0}=k_{B} T / D$. As in Ref. [4] we consider $D$ to the self-DC in a magnetized classical one-component plasma (OCP). From Eq. (5) we can relate $\mathcal{R}(\vartheta)$ to the DC through the DB relation. At vanishing damping $(\gamma \rightarrow 0)$, it can be shown that the parallel (to B) DC $D_{\|}$obtained from Eq. (5) and for $\vartheta=0$ coincides with the result of Ref. [9], where a kinetic equation method has been used. In particular, at $\gamma \rightarrow 0$, it is found that $\mathcal{R}_{0}(0) / \mathcal{R}_{\infty}(0)=D_{\infty, \|} / D_{0, \|}=2 / 3$ in agreement with Ref. [9]. Here $D_{\infty, \|}$ and $D_{0, \|}$ are the parallel self-DC at $B \rightarrow \infty$ and $B \rightarrow 0$, respectively. However at finite $\gamma$ and for $\vartheta=0$ the simple relation cited above is not obeyed in general, due to damping.

Fig. 1a,b shows $\mathcal{R}(\vartheta)$ given by Eq. (5), vs $\omega_{c} / \omega_{p}$ or $\gamma / \omega_{p}$. It is seen that the low-velocity SP increases with $\vartheta$ and $B$. Also the friction coefficient decreases with $\gamma$. It is opposite to the behavior found for an unmagnetized DEG [10]. A decrease of $\mathcal{R}(\vartheta)$ with $\gamma$ in the present case of a classical plasma is not attributable to the applied magnetic field because the field-free $\mathrm{FC} \mathcal{R}_{0}(\vartheta)$ shows a similar behavior. In a DEG an enhancement of the low-velocity SP with $\gamma$ is a quantum effect which is absent in our present study. For a DEG the domain of plasmon excitations is shifted towards smaller ion velocities [10] which increases the SP in this velocity regime. But in the present case the domain of collective excitations is shifted towards higher velocities and the friction coefficient decreases with $\gamma$.

The FC (6) may be compared with Ref. [3] where the friction coefficient in the collisionless plasma contains an anomalous term $\ln \left(v_{e} / v\right)$ vanishing at $\vartheta=0$. The physical origin of such an anomalous FC may be traced to the spiral motion of the electrons along $\mathbf{B}$. These electrons naturally tend to couple strongly with long-wavelength (small $k_{\|}$) and low-frequency (small $\omega=\mathbf{k} \cdot \mathbf{v}$ ) fluctuations along $\mathbf{B}$ provided by a slow projectile ion. Thus the contact time or the rate of energy exchange between the 
electrons and the fluctuations will be enhanced. At vanishing damping and in the limit of $v \rightarrow 0$, the frequency $\omega=\mathbf{k} \cdot \mathbf{v} \rightarrow 0$ tends to zero as well. The contact time $\sim \omega^{-1}$ thus becomes infinite and the friction coefficient diverges. The collisions of the plasma particles play a stabilizing role since the fluctuations provided by the slow ion are damped. Thus at $v \rightarrow 0$ the contact time is finite and is given by $\sim \gamma^{-1}$. As a result Eq. (6) does not contain a term like $\ln \left(v_{e} / v\right)$ but behaves as $\ln (1 / \gamma)$ at $\gamma \rightarrow 0$.

\section{KINETIC VERSUS HYDRODYNAMIC APPROACH}

We are looking for some contacts between our LR (kinetic) formulation and the previous hydrodynamic mode-coupling treatments based on the self-DC which is established via DB relation. In order to document the LIVSD highlighted by the DB relation, we first consider $B=0$ limit through the small $\epsilon \ll 1$ plasma parameter approximation for the self-DC [11]. Employing Eq. (5) and the DB relation one concludes that at $\gamma=0$ the self-DC obtained from these formulas coincides with [11] if $Z^{2}$ in $S$ is replaced by the quantity $P(Z)$, where $P(Z)=(Z+1 / \sqrt{2}) P_{1}(Z) / P_{2}(Z)$ with $P_{1}(Z)=32 Z^{2}+$ $75 \sqrt{2} Z+50, P_{2}(Z)=104 Z^{2}+111 \sqrt{2} Z+59$. For a proton $(Z=1)$ this factor is $P(1)=1.003$ and the agreement between both approaches is perfect. The factor $P(Z)$ accounts for the nonlinear coupling between an incoming ion and the surrounding plasma. However for highly charged ions $(Z \gg 1)$ this factor increases linearly with $Z, P(Z) \sim Z$, while more rigorous treatment shows that at strong ionplasma coupling the energy loss of an ion scales with $Z$ approximately like $Z^{1.5}$ [3].

Next, consider the case of a magnetized and collisional plasma. For simplicity, we consider electronproton plasma and a proton as a projectile particle. Exploring the moderately magnetized domain $\omega_{c} \geqslant$ $\omega_{p}$, one can elicit the field-free parallel and $B$-dependent transverse diffusions [12], $D_{0, \|}=3 \sqrt{\pi} v_{p}^{2} / \gamma_{c}$, $D_{0, \perp}=r_{L}^{2} \gamma_{c} / 3 \sqrt{\pi}$, where $v_{p}^{2}=k_{B} T / m_{p}, m_{p}$ is the proton mass, and $\gamma_{c}=\omega_{p} \epsilon \ln (1 / \epsilon)$ is the collision frequency, $r_{L}=v_{p} / \omega_{c}$. The transverse DC $D_{0, \perp}$ corresponds to a classical region, where $D_{0, \perp} \sim$ $B^{-2}$, and is valid for $\gamma_{c}<\omega_{c}<0.4 \omega_{p} Y(\epsilon)$ with $Y(\epsilon)=\left[\epsilon^{2} \ln (1 / \epsilon)\right]^{-1 / 2}$ [12]. At stronger magnetic field, $\omega_{c} / \omega_{p}>4 Y(\epsilon)$, one reaches the transverse hydro-Bohm regime with $D_{\perp} \sim B^{-1}$ featuring $D_{\perp}=D_{0, \perp}+\left(v_{p}^{2} / 2 \omega_{c}\right) \epsilon^{2}[\ln (1 / \epsilon)]^{3 / 2}$. For the intermediate plateau regime with $D_{\perp} \sim B^{0}$ between transverse DC given above, the diffusion coefficient valid at $0.4 Y(\epsilon)<\omega_{c} / \omega_{p}<Y(\epsilon)$ corresponds to $D_{\perp}^{\prime}=D_{0, \perp}\left[1+\left(0.6 \epsilon \gamma_{c} / \omega_{p}\right) \zeta^{2}\right]$ with $\zeta=\omega_{c} / \omega_{p}$ [12]. It is also important to stress that the quantitative predictions of the mode-coupling theory developed in Ref. [12] are strongly dependent on the values of the hydrodynamic cutoffs, which, in contrast to the kinetic theory, are introduced linearly. A reliable estimate of the magnetic field and plasma parameter dependence of the cutoffs have been obtained, but the numerical coefficients in the resulting DCs are not precisely known.

The friction coefficients $S(v) / v$ (at $v \rightarrow 0$ ) calculated with the help of the DB relation are shown in Fig. 1c, d. It is seen that there are no fundamental contradictions between kinetic and hydrodynamic approaches for the parallel case. Assuming a vanishing damping and magnetic field the ratio of the low-velocity SPs of both approaches is $S_{\|, \text {kin }} / S_{\| \text {,hyd }} \simeq \sqrt{2}$. But the numerical coefficients in the DCs are not precisely known. Thus including the numerical factor $\sqrt{2}$ into denominator of $D_{0, \|}$ the agreement between both approaches becomes complete. An apparently large discrepancy is documented for the transverse situation where $S_{\perp, \text { kin }} / S_{\perp \text {,hyd }} \sim[\epsilon \ln (1 / \epsilon)]^{\alpha} \ll 1$ and $\alpha$ varies between $2 \leqslant \alpha \leqslant 4$ depending on $B$. The kinetic regime seems to be restricted to $0.08=\gamma_{c} / \omega_{p}<\omega_{c} / \omega_{p}<0.4 Y(\epsilon)=10.1$ [12]. The discrepancy in the orthogonal case might be due to a different treatment of cutoffs in kinetic and hydrodynamic theories, i.e logarithmic vs linear. Actually the different hydro modes are normalized to distinct cutoffs. Upper hybrid ones are normalized to $1 / a_{e}$ while low frequency modes are normalized to $\gamma_{c} / v_{e}$. On the other hand at $\omega_{c} \gg \omega_{p}$, the only reasonable transverse cutoff should be $1 / a_{e}$ which, for instance, in the kinetic treatment is included as $\ln \left(1 / a_{e}\right)$. The basic physics involved in this orthogonal geometry pertains to kinetic theory when we rely on a collisional time while in the $B \rightarrow \infty$ limit leading to hydrodynamics, we incorporate the Larmor rotation of the charged particles, as well. 


\section{References}

[1] C. Cereceda, M. DePeretti, C. Deutsch, Phys. Plasmas 12, 022102 (2005)

[2] M. Tabak et al., Phys. Plasmas 1, 1626 (1994); C. Deutsch et al., Phys. Rev. Lett. 77, 2483 (1996)

[3] H.B. Nersisyan, C. Toepffer, G. Zwicknagel, Interactions Between Charged Particles in a Magnetic Field (Springer, Heidelberg, 2007)

[4] C. Deutsch, R. Popoff, Phys. Rev. E 78, 056405 (2008)

[5] J.W. Dufty, M. Berkovsky, Nucl. Instrum. Methods Phys. Res. B 96, 626 (1995)

[6] N.A. Krall, A.W. Trivelpiece, Principles of Plasma Physics (McGraw-Hill, New York, 1973)

[7] H.B. Nersisyan, C. Deutsch, A.K. Das, Phys. Rev. E 83, 036403 (2011)

[8] N.D. Mermin, Phys. Rev. B 1, 2362 (1970)

[9] J.S. Cohen, L.G. Suttorp, Physica A 126, 308 (1984); 123, 549 (1984)

[10] H.B. Nersisyan, A.K. Das, Phys. Rev. E 69, 046404 (2004)

[11] L. Sjögren, J.P. Hansen, E.L. Pollock, Phys. Rev. A 24, 1544 (1981)

[12] M.C. Marchetti, T.R. Kirkpatrick, J.R. Dorfman, J. Stat. Phys. 46, 679 (1987); 49, 871 (1987) 